\title{
Regulation of CD1d expression and function by a herpesvirus infection
}

\author{
David Jesse Sanchez,1,2,3 Jenny E. Gumperz, ${ }^{4}$ and Don Ganem ${ }^{1,3,5,6}$
}

\begin{abstract}
${ }^{1}$ George Williams Hooper Foundation, ${ }^{2}$ Biomedical Sciences Graduate Program, and ${ }^{3}$ Departments of Microbiology and Immunology, University of California, San Francisco, San Francisco, California, USA. ${ }^{4}$ Department of Medical Microbiology and Immunology, University of Wisconsin, Madison, Madison, Wisconsin, USA. ${ }^{5}$ Howard Hughes Medical Institute and ${ }^{6}$ Department of Medicine, University of California, San Francisco, San Francisco, California, USA.
\end{abstract}

\begin{abstract}
Little is known about the role of CD1d-restricted $\mathrm{T}$ cells in antiviral immune responses. Here we show that the lytic replication cycle of the Kaposi sarcoma-associated herpesvirus (KSHV) promotes downregulation of cell-surface CD1d. This is caused by expression of the 2 modulator of immune recognition (MIR) proteins of the virus, each of which promotes the loss of surface CD1d expression following transfection into uninfected cells. Inhibition of CD1d surface expression is due to ubiquitination of the CD1d $\alpha$-chain on a unique lysine residue in its cytoplasmic tail, which triggers endocytosis. Unlike MIR-mediated MHC class I downregulation, however, CD1d downregulation does not appear to include accelerated lysosomal degradation. MIR2-induced downregulation of CD1d results in reduced activation of CD1d-restricted T cells in vitro. KSHV modulation of CD1d expression represents a strategy for viral evasion of innate host immune responses and implicates $\mathrm{CD1d}$-restricted $\mathrm{T}$ cells as regulators of this viral infection.
\end{abstract}

\section{Introduction}

Recognition of a viral infection by the innate immune system is critical for both the efficient control of the earliest steps in the infection and the production of cytokine signals that activate the adaptive component of the immune response. In most infections (bacterial as well as viral), an innate response is normally activated in the hours immediately following infection and is initially cued by the production of generic signs of infection (e.g., doublestranded RNA, unmethylated CpG-containing polynucleotides, and LPS) and by pathogen-induced host cell signaling (e.g., via Toll-like receptors) (1). Virus-infected cells also typically release type I IFNs that can render surrounding cells less susceptible to viral infection and replication. Many cells of the innate immune system participate in the early response, most notably NK cells, which can recognize and kill infected cells and release abundant quantities of antiviral cytokines (2). Additionally, $\mathrm{T}$ cells that are restricted by CD1 molecules - a family of antigen-presenting molecules distantly related to class I molecules of the MHC - may also participate in early host responses (3).

Unlike MHC-encoded antigen-presenting molecules that present peptides, CD1 molecules have been found to present lipid and glycolipid antigens to T cells (4). Certain CD1 isoforms (i.e., CD1a, CD1b, and CD1c) have been shown to present pathogenspecific glycolipids (5-8). In contrast, it remains unclear whether the CD1d isoform presents foreign antigens, but this isoform has been shown to present self glycolipids to T cells $(9,10)$. CD1d chains are nonpolymorphic and are expressed only on a select number of cell types, including B cells, dendritic cells, hepatoctyes, and enterocytes (11). CD1d-restricted T cells appear to be evolu-

Nonstandard abbreviations used: EGFP, enhanced GFP; $\alpha$-GalCer, $\alpha$-galactosylceramide; iNKT cells, NK T cells expressing an invariantly rearranged TCR- $\alpha$ chain; KSHV, Kaposi sarcoma-associated herpesvirus; MIR, modulator of immune recognition; MVB, multivesicular body.

Conflict of interest: The authors have declared that no conflict of interest exists.

Citation for this article: J. Clin. Invest. 115:1369-1378 (2005).

doi:10.1172/JCI200524041. tionarily conserved, and comprise a population of $\mathrm{T}$ cells (known as NK T cells) that include those expressing an invariantly rearranged TCR- $\alpha$ chain (iNKT cells) as well as other T cells that utilize diversely rearranged TCRs $(11,12)$. The physiological ligands of CD1d-restricted T cells are unknown, but iNKT cells are strongly activated by a synthetic glycolipid called $\alpha$-galactosylceramide $(\alpha$-GalCer), which was originally derived from a marine sponge. This lipid has often been used as a surrogate antigen in experimental studies of iNKT cells and causes potent release of both IFN- $\gamma$ and IL-4 (13). CD1d-restricted T cells that use diversely rearranged TCRs do not appear to respond to $\alpha$-GalCer, and have not been as well studied as their iNKT cell counterparts.

Recently, the results of several studies have suggested possible roles for CD1-restricted $\mathrm{T}$ cells in the response to viral infection. For example, nonclassical (i.e., $\alpha$-GalCer-nonreactive) NK T cells are involved in the development of acute hepatitis in a transgenic mouse model of HBV infection (14). In addition, studies of respiratory syncytial virus, herpes simplex virus, coxsackievirus B3, and lymphocytic choriomeningitis virus (LCMV) have shown that the course of infection is altered in CD1d-deficient mice, suggesting possible involvement of CD1d in antiviral responses (15-17). However, virus-encoded ligands for CD1d have yet to be discovered, and the link between lipid recognition and viral infection has not been obvious. As a result, the role of CD1-restricted cells in antiviral defense has remained poorly understood.

One way to infer a biological role for a protein in antiviral immunity is to search for viral effectors that subvert the function of or impair expression of that protein. Herpesviruses have developed several methods of modulating the immune response directed against them, including the expression of proteins that block the transporter associated with antigen processing (TAP) peptide transporter, and a variety of proteins that impair MHC class I assembly, transport, or stability (reviewed in refs. 18, 19). The large number of redundant inhibitors of MHC class I suggests an important role for CTLs in defending against herpesvirus infection. Similarly, many herpesviruses encode proteins 
A

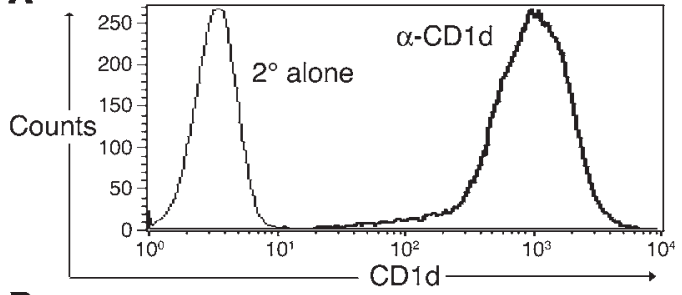

B
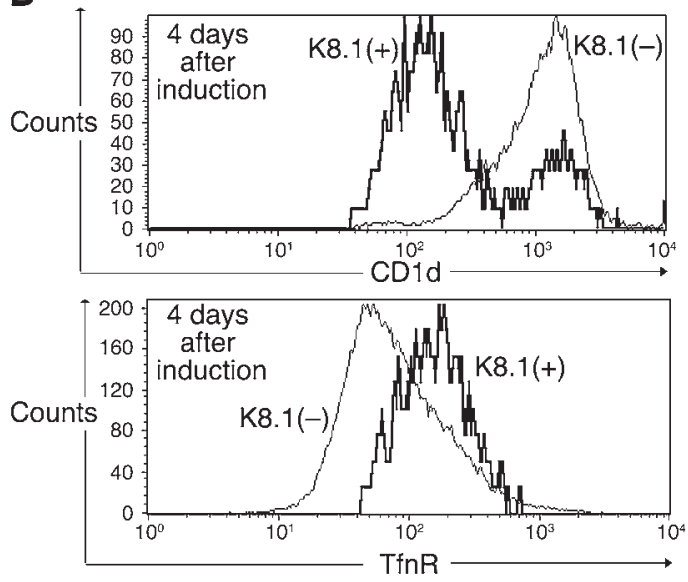

that impair type I IFN induction or action (20-23), and CMV has recently been found to encode proteins that block NK cellactivating receptors (24).

Kaposi sarcoma-associated herpesvirus (KSHV) is a B-lymphotropic herpesvirus that is the causative agent of at least 2 lymphoproliferative diseases (primary effusion lymphoma and multicentric Castleman disease) as well as Kaposi sarcoma, a tumor of endothelial origin (reviewed in refs. 25-27). Like other herpesvirus family members, it encodes proteins that block MHC class I expression on the cell surface (28-31). These are known as modulator of immune recognition (MIR) proteins; MIR1 and MIR2 are expressed during lytic replication and are encoded by the open reading frames K3 and K5, respectively. They function as membrane-bound ubiquitin ligases, leading to ubiquitination of their target proteins on lysine residues in their cytosolic tails (32). The result of this ubiquitination is the enhancement of the endocytosis of the target chain and its delivery (via the multivesicular body) to the lysosome, where it is proteolytically destroyed $(28,30)$. Both MIR1 and MIR2 reduce the cell surface levels of MHC class I; MIR2 also selectively targets the costimulatory molecules B7.2 and ICAM-1, both of which are involved in the enhancement of helper $\mathrm{T}$ cell signaling $(33,34)$. From these activities it can be inferred that both CTL and CD4 T cell action are likely to play important roles in host defense against KSHV. Here we show (a) that lytic KSHV infection also diminishes the surface expression of CD1d; (b) that both MIR1 and MIR2 can downregulate CD1d; and (c) that this downregulation strongly impairs NK T cell activation. The existence of a pathway for viral evasion of CD1drestricted $\mathrm{T}$ cell responses suggests a potentially important role for these $\mathrm{T}$ cells in the control of this herpesvirus infection.

\section{Results}

KSHV lytic replication decreases surface $C D 1 d$ levels. To investigate how KSHV infection affects CD1d expression, we analyzed CD1d cell surface expression on BCBL-1 lymphoma cells, a cell line that is

\section{Figure 1}

KSHV lytic replication decreases CD1d levels. (A) The levels of CD1d on uninduced $\mathrm{BCBL}-1$ cells, which are latently infected with KSHV. The background level of antibody staining of BCBL-1 cells is shown $\left(2^{\circ}\right.$ alone). (B) BCBL-1 cells were induced to enter the lytic replication cycle and allowed to proceed to 4 days after induction, after which the cells in the late portion of the lytic cycle could be monitored by K8.1 expression. The cells were stained with a rabbit primary antibody against K8.1 and an anti-rabbit secondary antibody, and with a Zenon-1APC-labeled antibody against CD1d and a PE-conjugated antibody against transferrin receptor (TfnR). The levels of CD1d for both the K8.1-positive [K8.1(+)] and K8.1-negative [K8.1(-)] populations were determined. To ensure that induction of lytic replication does not cause a general downregulation, the normal level of transferrin receptor was determined by analyzing a bulk population of K8.1-positive cells and creating a gate into which $80 \%$ of the cells fell. The K8.1-positive and K8.1-negative populations both showed similar levels of transferrin receptor; thus even in a nongated population, most cells had normal levels of cell-surface receptors.

latently infected by KSHV and normally expresses only a small subset of viral genes. Flow cytometric analysis of these cells showed that in their uninduced, latently infected state they express high levels of CD1d on their surface (Figure 1A). By treatment with phorbol esters, BCBL-1 cells were induced to enter the lytic replication cycle, whereupon a temporally regulated cascade of transcription occurs, ultimately resulting in expression of the vast majority of viral genes and the production of infectious viral progeny. Induction was generally inefficient, with only $5-20 \%$ of the cells expressing markers of lytic replication. In this study, entry into the lytic cycle was monitored by the expression of the virus-encoded cell surface glycoprotein K8.1 (35). By 4 days after lytic induction, flow cytometric analysis showed that the levels of CD1d were approximately 10 times lower in K8.1-positive cells than in the K8.1-negative cells that escaped induction (Figure 1B, top panel). This loss of surface CD1 was selective: K8.1-positive cells retained surface expression of the control transferrin receptor protein (in fact, transferrin receptor expression was modestly enhanced in this population; Figure 1B, bottom panel). Thus, lytically infected cells showed reduced CD1d cell-surface expression, and the loss of CD1d was not due to nonspecific toxicity resulting from the progression of lytic KSHV infection.

MIR1 and MIR2 induce CD1d downregulation. Because of the known ability of MIR1 and MIR2 to modulate immunologically relevant host cell surface proteins (e.g., MHC class I and B7.2), we investigated the effects of these viral proteins on CD1d expression. For these experiments, we employed fusion proteins in which MIR1 and MIR2 were fused to enhanced GFPs (EGFPs). We have previously shown that these chimeras are stably expressed and fully functional for downregulation of MHC class I chains upon either transient or stable transfection into cells (36). Accordingly, each chimera was transfected into BJAB cells, which express low levels of CD1d on their surface. By gating on EGFP-positive cells, we could identify cells expressing MIR, and the levels of CD1 in these cells were quantitated by flow cytometry. As shown in Figure 2A (top left panel), both MIR1- and MIR2-transfected cells showed a marked decrease in levels of CD1d on the cell surface, down to a level comparable with nonstained BJAB cells. The data shown in Figure 2A (middle left panel) confirms that both MIR-EGFP fusions were able to downregulate MHC class I expression in these cells. Note that the surface levels of HLA-DR, a representa- 
A
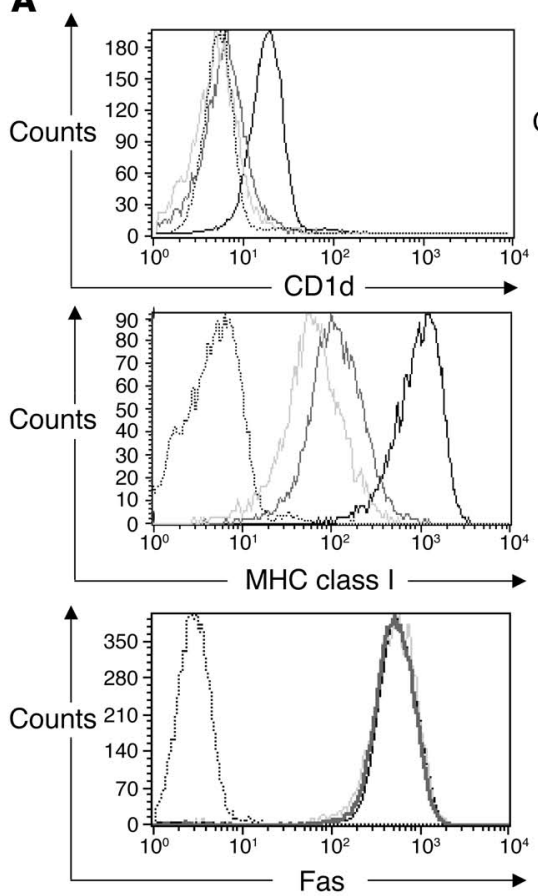
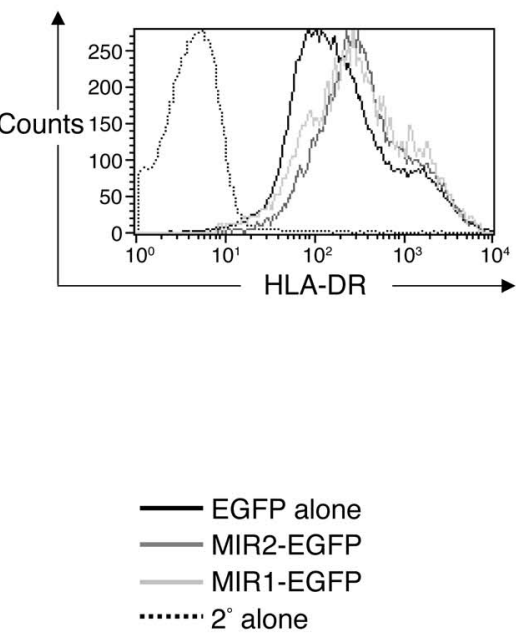

B
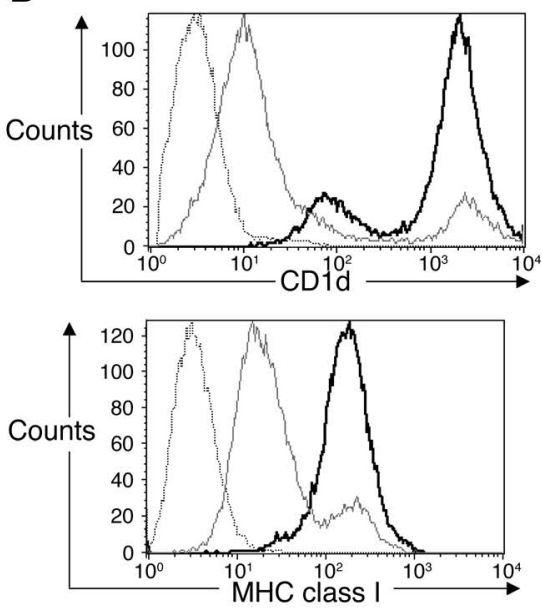

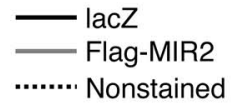

Figure 2

MIR1 and MIR2 induce CD1d downregulation. (A) BJAB cells were transiently transfected by electroporation with expression vectors for EGFP, MIR1-EGFP, or MIR2-EGFP. Thirty-six hours after transfection, the cells were stained with Zenon-1-APC-labeled mouse mAbs against human CD1d. Levels of HLA-A, HLA-B, and HLA-C (MHC class I); HLA-DR (MHC class II); CD1d; and Fas are shown. For reference, the nonstained control population is also shown. (B) HepG2 cells, which express a high level of endogenous CD1d, were stably transduced with retroviral vectors encoding either lacZ or Flag-tagged MIR2. A mixed population of stable cells was selected, stained with Zenon-1-APC-labeled mAbs, and analyzed by flow cytometry. As a reference, the histogram for the nonstained control is also shown.

tive MHC class II molecule, and Fas are both unchanged in cells expressing MIR1 and MIR2 (Figure 2A, top right and bottom left panels, respectively). Thus, CD1d downregulation is selective and is not an indirect consequence of alterations in MHC class II delivery to the cell surface - an important result given earlier work implicating the MHC class II biosynthetic pathway in the proper trafficking of CD1d to the cell surface (37).

The low level of surface CD1d on BJAB cells limits the dynamic range of the CD1d reduction assay and may lead to underestimation of the magnitude of the inhibition. Therefore, HepG2 hepatoblastoma cells, which have high levels of endogenous CD1d on their surface, were selected for further study. HepG2 cells were transduced with a retrovirus encoding a functional, Flag-tagged MIR2 and neomycin resistance marker, and neoresistant HepG2 cells were selected as a bulk population. As a control, a similar population was selected following transduction with a lacZ/neo retrovirus. Each cell population was then analyzed by flow cytometry. As shown in Figure 2B, left panel, the levels of CD1d were reduced up to 100 -fold in the MIR2 stable cells compared with those in control HepG2 cells that stably express lacZ. This is an even greater reduction than was observed on $\mathrm{MHC}$ class I chains in the same populations of cells (Figure 2B, right panel). Thus, the effect of MIR2 on CD1d appears very robust. Attempts to generate stable transductants of either HepG2 or BJAB with MIR1 were unsuccessful, as the resulting transductants rapidly lost their initially high level of expression of MIR1. Nonetheless, transient expression of MIR1-EGFP indicates that it possesses substantial ability to downregulate CD1d (Figure 2A). Thus, CD1d regulation, like MHC class I but unlike B7.2 and ICAM-1 downregulation (33, 34), has been conserved in both MIR family members.

MIR2 downregulation of CD1d reduces $C D 1$-restricted $T$ cell activation. The effect of MIR-mediated downregulation of CD1d on the activation of CD1d-restricted T cells was investigated in an in vitro coculture system. In this system, BJAB cells that stably expressed either lacZ or MIR2 were incubated with CD1drestricted NK T cell clones in the presence of $\alpha$-GalCer, and IFN- $\gamma$ release was measured by ELISA as previously described (9). As shown in Figure 3A, CD1d-restricted T cell clones incubated with MIR2-expressing BJAB cells showed reduced IFN- $\gamma$ production compared with that of clones incubated with the lacZexpressing BJAB cells. This effect was consistent across 3 different CD1d-restricted T cell clones. Next, IFN- $\gamma$ release induced by the MIR2- and lacZ-transfected BJAB cells was monitored in the presence and absence of $\alpha$-GalCer. As expected, IFN- $\gamma$ production by the $\mathrm{T}$ cell clones was greatly increased in the presence of $\alpha$-GalCer compared with that in its absence (Figure 3B). Also, while MIR2 expression led to a marked reduction in IFN- $\gamma$ secreted in response to $\alpha$-GalCer, it did not dramatically change the levels of IFN- $\gamma$ produced by the $T$ cells in the absence of $\alpha$-GalCer (Figure 3B). We also examined the effects of anti-CD1d antibodies on activation. As shown in Figure 3C, anti-CD1d reduced activation by control $\mathrm{BJAB} / \mathrm{lac} Z$ cells to an extent similar to that caused by the expression of MIR2. Addition of antiCD1d to BJAB/MIR2 cells in the assay further reduced the level 


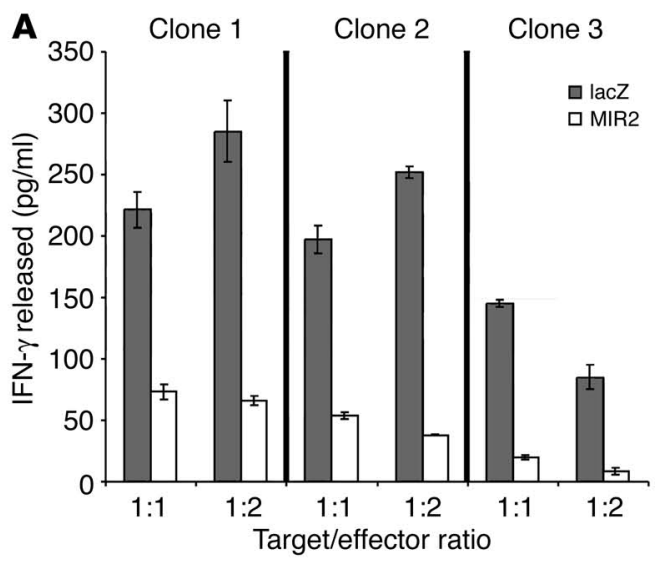

B

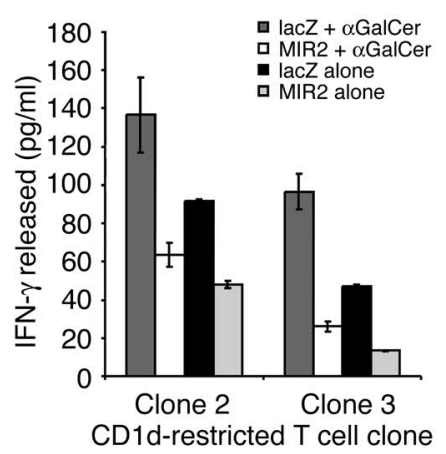

C

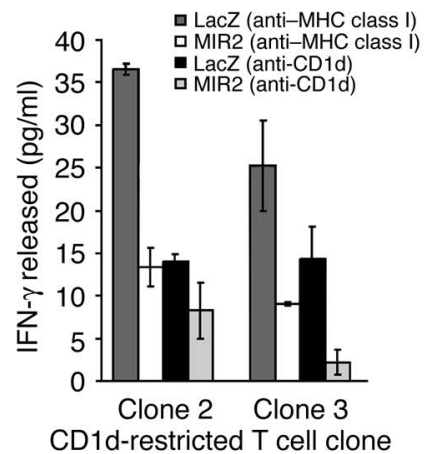

Figure 3

MIR2 downregulation of CD1d lowers CD1d-restricted T cell activation. (A) BJAB cells stably transduced with either lacZ (gray bars) as a control or MIR2 (white bars) were cocultured with CD1d-restricted T cells. This coculture was done in the presence of $\alpha$-GalCer to provide an activating ligand for the T cells. Each coculture was performed in triplicate. After 18 hours of coculture, each supernatant was assayed for IFN- $\gamma$ release by ELISA. (B) As in A, coculture experiments were done with CD1d-restricted T cells and BJAB cells, this time either in the presence or absence of $\alpha$-GalCer. Cocultures were done with $\alpha$-GalCer lacZ (light gray bars), $\alpha$-GalCer MIR2 (white bars), lacZ without $\alpha$-GalCer (black bars), and MIR2 without $\alpha$-GalCer (dark gray bars). After 18 hours, the supernatants were recovered, and the IFN- $\gamma$ in each supernatant was assayed by ELISA. (C) As in A, coculture experiments were done with CD1d-restricted T cells and BJAB cells, either in the presence of mouse anti-MHC class I as a control (lacZ, dark gray bars; MIR2, white bars) or mouse anti-CD1d (lacZ, black bars; MIR2, light gray bars). After 18 hours, the supernatants were recovered, and the IFN- $\gamma$ in each supernatant was assayed by ELISA. Results are shown as means \pm SD.

of activation seen in this context, indicating the presence of a low level of residual CD1d on the surface of these cells. Thus, MIR2 expression sharply reduces but does not entirely eliminate residual CD1d-dependent signaling.

MIR2 induces ubiquitination and internalization of CD1d and/or CD1d-associated proteins. In earlier studies of MIR-mediated downregulation of MHC class I and B7.2, we (32) and others (38) showed that downregulation of the target proteins is dependent upon the ubiquitination of cytosolic lysine residues in the target. Ubiquitination triggers internalization of these chains and directs the nascent endosome into the multivesicular body (MVB) pathway (39), where its contents are sorted to lysosomes and degraded by lysosomal proteases $(28,32)$. Although not all of the steps involved in this sorting pathway are fully understood, it is known that ubiquitin also plays prominent roles in these processes. For example, many components of the MVB machinery are either ubiquitinated or harbor ubiquitin-binding motifs (reviewed in ref. 40).

Given the importance of ubiquitination in MIR activity, we asked whether CD1d or chains associated with it undergo ubiquitination in an MIR-dependent fashion. HepG2 cells stably transduced with either lacZ or MIR2 were lysed in detergent; CD1d was immunoprecipitated, separated by SDS-PAGE, transferred to solid supports, and immunoblotted with anti-ubiquitin conjugat-

\section{Figure 4}

MIR2 expression leads to the coimmunoprecipitation of ubiquitinated proteins with CD1d. HepG2 cells stably transduced with either lacZ or MIR2 were lysed, and human CD1d (hCD1d) was immunoprecipitated from the lysates. The immunoprecipitate was then separated by SDSPAGE and transferred to nitrocellulose, and the presence of ubiquitinated proteins was determined by Western blotting with an HRP-conjugated anti-ubiquitin antibody (Anti-Ub-HRP). As shown, only in the presence of MIR2 did ubiquitinated proteins coimmunoprecipitate with CD1d. ed to HRP. As shown in Figure 4, immunoprecipitation with CD1d induces a strong and heterogeneous ubiquitination signal only in MIR2-expressing cells and not in lacZ-expressing control cells. As human CD1d has been shown to form stable complexes with other proteins (i.e., MHC class II) (37), the ubiquitinated bands in Figure 4 may represent multiply ubiquitinated CD1d chains, ubiquitination of multiple associated proteins, or both.

MIR2 induces CD1d downregulation, and ubiquitination of CD1d is dependent on intracytoplasmic lysines. The CD1d intracytoplasmic tail contains a single lysine, which may be a target of the MIR ubiquitin ligases (Figure 5A). In a previous study, the removal of lysines from the cytoplasmic tail of potential MIR downregulation targets abolished the ubiquitination of the target proteins and their subsequent downregulation (32). Interestingly, murine CD1d has no cytosolic lysines and is not downregulated by MIR2 in murine cells or even when expressed in human CD1d-positive cells (see Supplemental Figure 1; available online with this article; doi:10.1172/JCI200524041DS1). Addition of a lysine into the cytoplasmic tail of mouse CD1d also

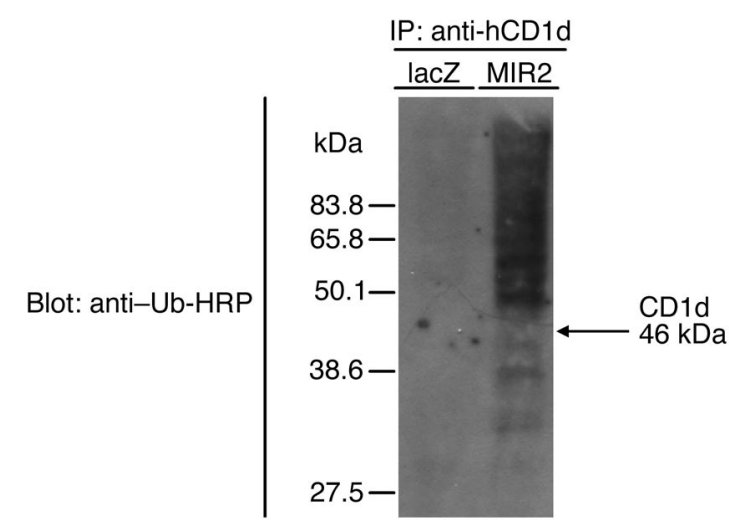


A

Human CD1d (WT)
Human CD1d $(\mathrm{K} \rightarrow \mathrm{R})$

B

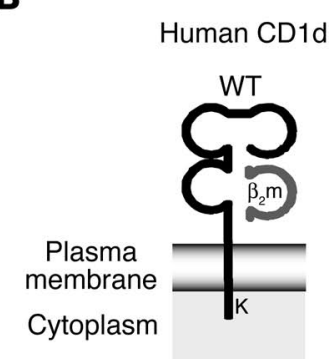

Extracellular domain domain

... SY T SMGLIALAVLAVLLFLLIVGFT SRFKR QTSY QGVL ... SYTSMGLIALAVLAVLLFLLIVGFTSRFRRQTSYQGVL

Mouse extracellular/human transmembrane and intracellular chimeric CD1d

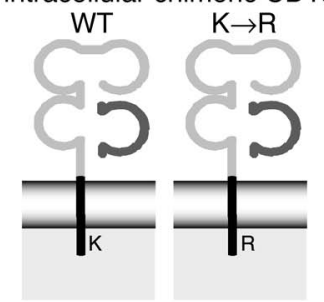

C

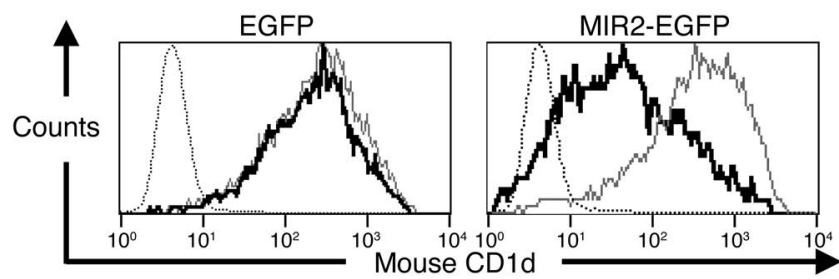

Transfected chimeric CD1d

D

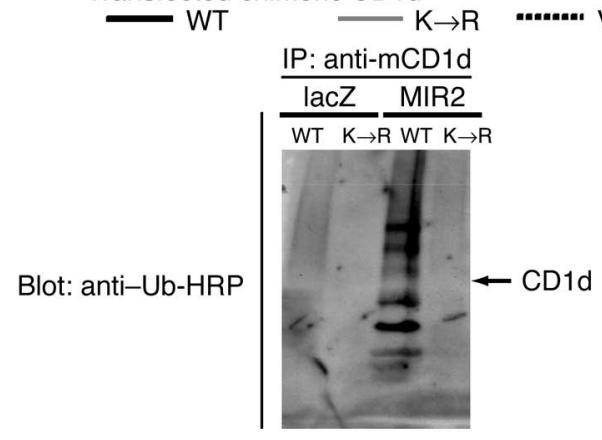

Intracytoplasmic tail

R R 
A

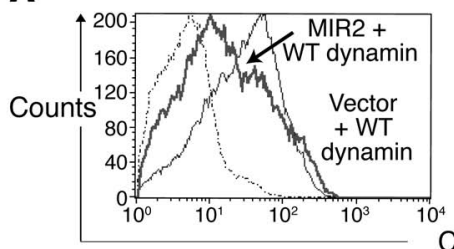

C

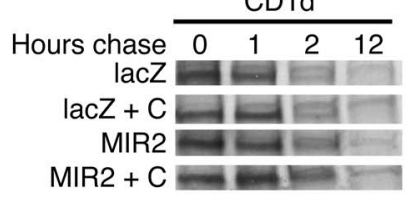

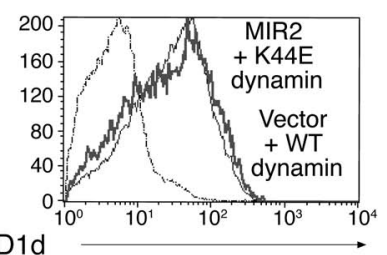

MHC class I

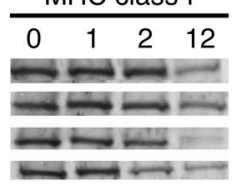

D

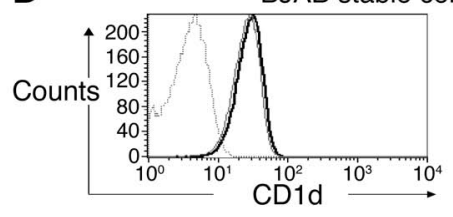

BJAB stable cells, total cellular levels
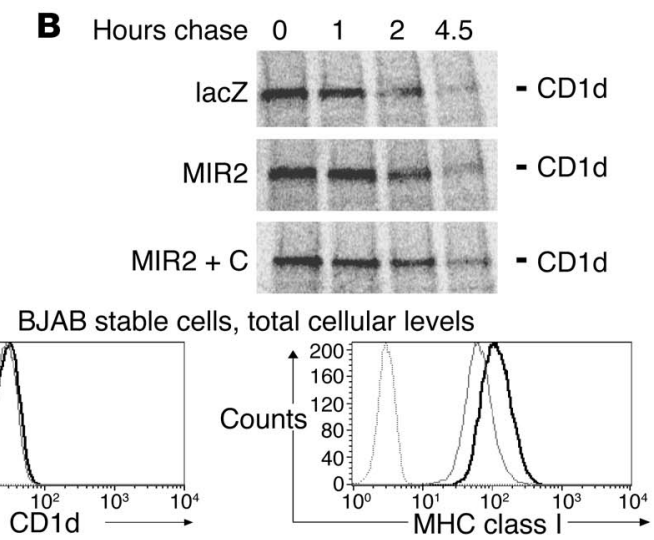
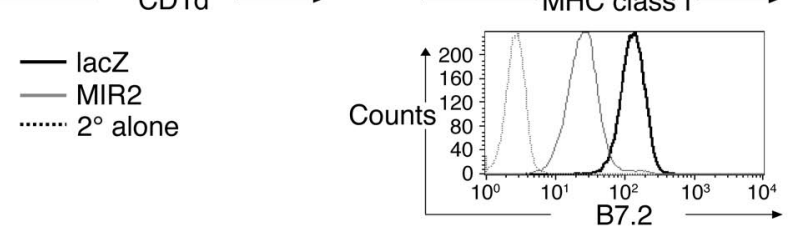

Figure 6

MIR2 downregulation of CD1d is due to endocytosis but not enhanced degradation. (A) BJAB cells were transiently transfected with wild-type chimeric CD1d. Both panels show the level of chimeric CD1d when cotransfected with wild-type dynamin (solid black lines) as well as the level of CD1d antibody background cross-reactivity (dotted black lines). The gray lines represent chimeric CD1d levels in the presence of MIR2 and either wild-type (left panel) or dominant-negative dynamin (K44E, right panel). (B) The half-life of CD1d in BJAB cells expressing lacZ or MIR2 was analyzed by pulse chase. As shown, the presence of the MIR2 gene does not change the half-life of the CD1d chains. Chloroquine (C) was added to block lysosomal degradation and showed no effect. (C) The half-life of CD1d in HepG2 cells expressing lacZ or MIR2 were analyzed by pulse chase. As shown, the presence of the MIR2 gene does not change the half-life of the CD1d chains (left blots), but does increase the levels of degradation of MHC class I (right blots). Addition of chloroquine stopped the MHC class I degradation. (D) Total cellular levels of proteins in BJAB cells that were stable for expression of MIR2 or lacZ was determined by flow cytometry. The presence of the MIR2 gene did not change the total levels of CD1d, even though the surface levels of CD1d were decreased. This is in contrast to the decreased total and surface levels of B7.2 and MHC class I.

dynamin, whose activity is required for endosome formation (41). BJAB cells were transiently transfected with the wild-type CD1d chimera and either a vector control or MIR2. In addition, each culture received a vector expressing either a wild-type or dominantnegative mutant dynamin, and the IRES-dependent expression of EGFP in a bicistronic message. Using flow cytometry, we then analyzed the GFP-expressing (i.e., transfected) cells for cell-surface CD1d. As expected, MIR2 expression in the presence of wild-type dynamin led to strong downregulation of CD1d (Figure 6A, left panel). However, when the dynamin dominant-negative mutant K44E was expressed, this downregulation was completely abrogated (Figure 6A, right panel). This effect was dependent upon MIR2 expression, as K44E expression had little impact on surface CD1d in the absence of MIR2 (data not shown). Thus, the disappearance of cell-surface CD1d upon MIR2 expression appears to be due to internalization via endocytosis.

MIR-mediated downregulation of MHC class I induces a rapid degradation of the MHC class I chains in a lysosome-dependent manner (28). To examine the half-life of CD1d chains in the presence of the MIR proteins, we carried out a pulse-chase experiment to track the metabolism of newly synthesized CD1d polypeptides. BJAB (Figure 6B) and HepG2 cells (Figure 6C) expressing either lacZ or MIR2 were labeled for 20 minutes with $S^{35}$ trans-label and chased in unlabeled medium for 1,2, or 4.5 hours in the case of the $\mathrm{BJAB}$ cells or 12 hours in the case of the HepG2 cells; then, CD1d chains were immunoprecipitated and examined by SDS-PAGE. Comparison of the profiles of CD1d in MIR2- and lacZ-expressing cells revealed no clear change in the half-life of the proteins in the presence of MIR2 in either cell type (Figure 6B and Figure 6C, left panel). Moreover, the addition during the chase of chloroquine, an inhibitor of lysosomal acidification and proteolysis during the chase failed to lead to substantial accumulation of labeled CD1d chains (Figure 6B, rows 2 and 3, and Figure 6C, left panel, rows 3 and 4), though it did partially rescue the appearance of MHC class I chains under these conditions (Figure 6C, right panel). Thus, in contrast to MHC class I, CD1d is ubiquitinated and removed from the cell surface in the presence of MIR2, but does not undergo enhanced degradation. Correspondingly, flow cytometric analysis of steady-state total CD1d levels (as measured by antibody staining in saponin-permeabilized cells) revealed no appreciable change in the level of CD1d, irrespective of the presence or absence of MIR2 (Figure 6D). As expected, however, total B7.2 and MHC class I levels were both lower in the MIR2 cells than in the control cells.

\section{Discussion}

Our finding that cell-surface CD1d is downregulated during lytic KSHV infection and by expression of KSHV MIR proteins represents the first report to our knowledge of modulation of the CD1d system by a virus and suggests a role for this system in the control of KSHV infection. We propose that CD1d downregulation may assist KSHV during the early phases of a new infection by impairing the local activation of CD1d-restricted T cells, thereby (a) reducing the direct release of antiviral cytokines (e.g., IFNs) into the microenvironment in which viral replication has begun, and (b) reducing the signals that promote a subsequent adaptive immune response to the virus. This would be analogous to roles 
we have previously proposed for MIR2 based on its abrogation of CD4 T cell activation through downregulation of B7.2 (33). In addition, recent studies have suggested that immediately after infection, but before the establishment of long-term latency, a small amount of MIR2 may be expressed and contribute to rapid reduction of cell-surface MHC class I and possibly CD1d during the initial establishment of latency (42). Unfortunately, the inability to infect mice or other experimental animals with KSHV (and the fact that murine CD1d cannot be downregulated by the MIRs, as shown in Supplemental Figure 1) make direct in vivo tests of these predictions impossible at present.

$\mathrm{KSHV}$ is not the only virus to modulate the innate immune system. Recent studies of CMV have focused on the selective modulation of NK cell-activating receptors by viral proteins $(24,43)$. This is thought not only to impair NK cell-mediated lysis of infected cells, but also to delay NK cell activation of the adaptive response and subsequent polarization of the helper T cell response (44). CD1drestricted $\mathrm{T}$ cells or NK $\mathrm{T}$ cells may represent a system parallel to the activation of NK cells for the rapid detection of viral infection and the enhancement of adaptive immunity. Blocking activation of CD1d-restricted T cells may also modulate the activation of NK cells (45). If so, KSHV may achieve indirectly what CMV achieves by direct blockade of NK-activating receptors on infected cells.

Many questions remain to be answered concerning the role of the CD1d system in the control of a viral infection. Chief among them are those regarding: (a) the determination of the ligand presented by CD1d during a viral infection; and (b) the way in which CD1d-restricted T cells become specifically activated. While it is not clear whether the physiological ligands of CD1drestricted $\mathrm{T}$ cells include foreign compounds, many CD1drestricted $\mathrm{T}$ cell clones seem to be autoreactive against lipids of host origin $(10,46)$. It has recently been shown that this reactivity to self antigens can be exploited in a novel activation strategy that may allow for the rapid triggering of CD1d-restricted T cells in a wide variety of pathogenic infections (9). However, other lipids derived from tumors may be inhibitory in nature, which suggests multiple systems of control within the CD1d-restricted $\mathrm{T}$ cell system (47). Under normal circumstances, CD1d-restricted $\mathrm{T}$ cell recognition of self antigens results in little cytokine secretion; however, in the presence of the cytokines IL-12 and IL-2, which are produced by APCs in response to microbial products, the effect of self-antigen recognition is amplified, leading to potent IFN- $\gamma$ production by CD1d-restricted T cells. This mechanism seems to be responsible for the early activation of CD1d-restricted $\mathrm{T}$ cells observed in vivo in a bacterial infection (9) and may be sufficiently generic to allow for the activation of CD1d-restricted T cells in viral infections. Alternatively, viral infections may trigger the production of new classes of self lipids that are part of a stress-activated system; these lipids could then be introduced into the CD1d antigen-processing pathway. For example, as a result of viral infection, normal glycolipids could become differently glycosylated or different types of lipids could gain access to CD1d-antigen loading sites, leading to the presentation of those modified lipids (i.e., altered self) on infected B cells. Uninfected APCs might also acquire and present these lipids following endocytosis of cell membranes from lysed host cells or phagocytosis of infected or apoptotic cells. A third possibility is that viruses like KSHV might encode either myristoylated or palmitoylated proteins whose lipidated peptide fragments might be presented in the hydrophobic binding pock- et of CD1d. Although only fragmentary and controversial evidence links the CD1d system to peptide presentation (48), direct presentation of viral lipopeptides remains a formal possibility.

Interestingly, whereas we observed a marked effect of MIR2 expression on CD1d-restricted $\mathrm{T}$ cell activation in response to the exogenously added antigen $\alpha$-GalCer (which presumably reflects the decreased surface expression of CD1d in the presence of MIR2), the modest IFN- $\gamma$ secretion obtained in the absence of $\alpha$-GalCer (presumably the response of the CD1d-restricted T cells to self antigens presented by APCs) was not significantly reduced. Further investigation will be required to determine the cause as well as the significance of this difference; however, we suspect it could be due to a differential effect of the MIR proteins on CD1d loading of endogenously versus exogenously derived antigens. CD1d molecules traffic extensively through the endocytic system (49-53) and are capable of presenting both exogenously derived (54) and endogenously derived antigens $(46,55)$. However, it is not clear in which compartments the relevant physiological ligands of CD1drestricted T cells are loaded, and it is possible that exogenous and endogenous cellular lipids may be loaded at different sites. If so, MIR proteins may direct CD1d polypeptides to compartments in which exogenous lipids are not loaded, but endogenous ones are.

Our results also shed light on how the MIR proteins influence the trafficking of molecules involved in immune recognition. In all cases, the target proteins are ubiquitinated and endocytosed. In the case of MHC class I chains, enhanced endocytosis results in chains being delivered via the MVB to lysosomes for degradation. Since the MVB sorting steps also prominently involve ubiquitinated proteins, it has been suggested that MIRs may have additional ubiquitination targets downstream of the endocytic step $(39,40)$. However, in the case of CD1d, we found little evidence for enhanced lysosomal delivery of the protein, despite its efficient endocytosis under the influence of the MIR proteins. This decoupling of ubiquitin-mediated endocytosis and degradation by the MIR proteins indicates that the primary MIR-regulated step is endocytosis. Perhaps the difference between CD1d and MHC class I reflects the different pathways normally taken by these proteins. Murine CD1d has a long half-life and is known to be actively recycled from internal vesicles to the plasma membrane; indeed, at steady state much of the cellular complement of the protein resides in internal vesicles (56). This suggests that lysosomal degradation plays at best a modest role in the turnover of CD1d polypeptides. Thus, in the case of both CD1d and MHC class I, the simplest explanation for MIR action is that it directly targets the endocytic step. When the protein is naturally sorted to degradative lysosomes (e.g., MHC), this results in enhanced turnover; when the protein can sort to the lysosome and not be degraded (e.g., CD1d), the result is enhanced internalization. By carefully analyzing the complement of ubiquitinated host proteins that complex with MHC class I and CD1d in the presence of MIR2, we hope to be able to dissect the biochemical details of the downregulatory mechanism and thus shed further light on the trafficking of these important mediators of immune recognition.

\section{Methods}

Cell culture. BCBL-1, an established KSHV-infected human B cell line free of EBV infection, was grown in RPMI with glutamine, sodium bicarbonate, and $\beta$-mercaptoethanol (Invitrogen Corp.). HepG2, a hepatocellular carcinoma cell line, was grown in minimal essential medium (Invitrogen Corp.). Phoenix, a 293T-based retroviral packaging cell line, was grown in 
DMEM, and the BJAB B cell lymphoma cell line was grown in RPMI medium 1640. All media were additionally supplemented with $10 \%$ (vol/vol) fetal calf serum as well as a combination of penicillin and streptomycin. $\mathrm{BJAB}$ cells were transfected by electroporation $(250 \mathrm{~V}, 950$ microfarads) of $20 \mu \mathrm{g}$ of plasmid DNA into $10^{7}$ cells in $0.5 \mathrm{ml}$ serum-free medium. The cells were then transferred to complete RPMI, which was preincubated to $37^{\circ} \mathrm{C}$. The transfection efficiency of BJAB cells was routinely $30-50 \%$ under these conditions (36).

Stable BJAB and HepG2 cells expressing MIR proteins were created by retroviral transduction. Upon transfection with the retroviral vectors (pBMN or pBMP, containing either neomycin resistance or puromycin resistance), the Phoenix packaging cell line produces replication-defective viral particles that were used to stably transduce recipient HepG2 or BJAB cells. Transient transfections of Phoenix cells were performed using Fugene6 (Roche Applied Science) according to the manufacturer's suggested protocol. Phoenix cells were transfected, media was changed 24 hours after transfection, and the virus-containing supernatant was harvested 48 hours after transfection, filtered through a $0.45-\mu \mathrm{m}$ syringe filter, and diluted with Polybrene (final dilution, $4 \mu \mathrm{g} / \mathrm{ml}$ ). HepG 2 and BJAB cells were infected by spin infection $\left(800 \mathrm{~g}\right.$ for 2 hours at $22^{\circ} \mathrm{C}$ ) using $2 \mathrm{ml}$ of viral supernatant per well of a 6-well dish. Selection of transduced HepG2 or BJAB was started 36 hours after infection by adding $1.5 \mathrm{mg} / \mathrm{ml}$ and $0.8 \mathrm{mg} / \mathrm{ml}$ of G418 to $\mathrm{BJAB}$ and HepG 2 cells, respectively, or $1 \mu \mathrm{g} / \mathrm{ml}$ of puromycin to BJAB cells. All cell lines were selected and used as pooled populations.

Derivation of CD1d-restricted T cell clones. CD1d-restricted T cell clones were derived by staining human PBMC with $\alpha$-GalCer-loaded human CD1d tetramers as previously described (57). Positively stained T cells were singlecell sorted by flow cytometry and cultured in the presence of irradiated allogeneic human PBMC in RPMI culture medium containing $10 \%$ heatinactivated fetal bovine serum, $1 \%$ human $\mathrm{AB}$ serum, $1 \%$ penicillin combined with streptomycin (Invitrogen Corp.), $2 \mathrm{mM}$ L-glutamine (Invitrogen Corp.), $1 \times$ PHA, and $2 \mathrm{nM} \mathrm{IL-2}$. Cloned T cell lines were expanded in culture using the same medium but lacking PHA and were restimulated periodically by exposure to irradiated allogeneic PBMC in the presence of PHA.

Coculture and IFN release assays. Cocultures were designed with $10^{4}$ target cells and recently stimulated CD1d-restricted T cells in target-to-effector ratios of $1: 1$ or 1:2. These cells were cocultured in 96-well dishes for 18 hours at $37^{\circ} \mathrm{C}$, after which the supernatants were removed and the levels of IFN- $\gamma$ were analyzed. DMSO-dissolved $\alpha$-GalCer was kept at $4^{\circ} \mathrm{C}$ at a concentration of $100 \mu \mathrm{g} / \mathrm{ml}$. It was sonicated at $37^{\circ} \mathrm{C}$ in a water bath sonicator before being used at a concentration of $50 \mathrm{ng} / \mathrm{ml}$. Quantitation of IFN- $\gamma$ production was determined by averaging triplicate wells and using linear regression from a standard curve and determined by a standard ELISA using a BD Biosciences OptEIA Set.

Construction of chimeric CD1d expression vectors. Mouse CD1d (catalog no. 948632 VK35G05R'; American Type Culture Collection) was PCR amplified with the following primers: forward, 5 '-GTACTGGATCCGCCGCCACCATGCGGTACCTACCATGGCTGTT-3'; reverse, 5'-TGCATGCGGCCGCTCACCGGATGTCTTGATAAGCGCTTCTCCTTCTCCAGATATAGTAGACTACAGCACCC-3'; using PfuUltra (Stratagene) per the manufacturer's instructions. The fragment was cloned into the BamHI and NotI sites of PCR3.1 (BD Biosciences - Clontech). Human CD1d (Mammalian Gene Collection no. 34622; IMAGE no. 5229534) was PCR amplified with the following primers: forward, 5'-ATCATGGATCCGCCGCCACCATGGGGGTGCCTGCTGTTTCTGCTG-3'; reverse (wild-type), 5'-TATATGCGGCCGCTCACAGGACGCCCTGATAGGAAGTT-3'; reverse (lysine-to-arginine mutant), 5'-TATATGCGGCCGCCACAGGACGCCCTGATAGGAAGTTTGCCTGCGAAACCGGGAGGTAAAGCCCACAATGAGG-3'. All products were recovered and cloned into the BamHI and NotI sites of pCR3.1 (BD Biosciences - Clontech). Chimeric CD1d was generat- ed by creating fragments of the extracellular domain of mouse CD1d with the mouse CD1d forward primer and the reverse primer 5'-CAGGACTGCCAAGGCAATCAAGCCCATGGGTGCTTGCCTGGCATCCC-3' and of the transmembrane and cytoplasmic tail of human $\mathrm{CD} 1 \mathrm{~d}$ with the reverse primers for human CD1d (either wild-type or lysine-to-arginine mutant) and the forward primer 5'-GGGATGCCAGGCAAGCACCCATGGGCTTGATTGCCTTGGCAGTCCTG-3' with PfuUltra (Stratagene) according to the manufacturer's directions. The fragments were gel purified and mixed in single PCR reaction with the forward primer for full-length mouse CD1d and the reverse primer for full-length human CD1d (either wild type or lysine-to-arginine mutant) using Vent DNA Polymerase (New England Biolabs) according to the manufacturer's directions. The chimeric fragments were recovered and cloned into the BamHI and NotI sites in PCR3.1 for expression studies or subcloned into pMX-PIE (a gift from M. Lodoen and L.L. Lanier, UCSF, San Francisco, California, USA) for creation of retroviruses for stable transduction.

Flow cytometry. BCBL-1 cells were fixed in $4 \%$ paraformaldehyde and washed 3 times with PBS. The cells were then blocked with a wash of PBS plus $1 \%$ BSA. BCBL-1 cells were then incubated with rabbit anti-K8.1 in $100 \mu \mathrm{l}$ of PBS plus $1 \%$ BSA for 45 minutes on ice, washed with PBS plus $1 \%$ BSA, incubated with mouse anti-rabbit conjugated to FITC (SigmaAldrich) in $100 \mu \mathrm{l}$ PBS plus 1\% BSA, and washed with PBS plus 1\% BSA. The cells were then further incubated with a mixture of PE-conjugated mouse anti-transferrin receptor (Caltag Laboratories) and mouse antiCD1d (Clone NOR3.2; Calbiochem, EMD Biosciences) labeled with Zenon Allophycocyanin Mouse IgG1 Labeling Kit (Zenon-1-APC; Invitrogen Corp.) in $100 \mu \mathrm{l}$ of PBS plus 1\% BSA. After this last incubation, the cells were washed extensively with PBS and resuspended in $200 \mu$ l of PBS for analysis by a FACScalibur (BD Biosciences) flow cytometry system.

For BJAB and HepG2 experiments, cells were washed once with PBS plus $1 \%$ BSA and incubated without fixation with the appropriate antibody on ice. CD1d was analyzed by using mouse anti-CD1d antibody (Clone NOR3.2; Calbiochem, EMD Biosciences) that was labeled with Zenon-1-APC (Invitrogen Corp.). Extracellular mouse CD1d was analyzed by rat-derived anti-mouse CD1d antibodies (Clone 1B1, 1:100 dilution; BD Biosciences - Pharmingen) with a goat-derived anti-rat secondary antibody coupled with allophycocyanin (1:100 dilution; Caltag Laboratories). In addition, mouse anti-human MHC class I (Clone W6/32) precoupled to PE (Caltag Laboratories) was used at a dilution of 1:100 in PBS plus 1\% BSA in these experiments. After incubation and extensive washings in PBS, cells were analyzed by flow cytometry with a FACScalibur flow cytometry system.

Coimmunoprecipitation and Western blotting. Stable HepG2 cells or $5 \times 10^{6}$ $\mathrm{BJAB}$ cells contained in a confluent 10-cm dish were washed 3 times with ice-cold PBS, and $1 \mathrm{ml}$ of ice-cold PBS plus 1\% Igepal (NP-40 type detergent; Sigma-Aldrich) was added to the dish or tube. The cells were suspended in the lysis buffer and passed twice through a 21-gauge syringe, and the lysate was cleared by spinning in a microcentrifuge for 10 minutes at maximum speed $(13,000 \mathrm{~g})$ at $4^{\circ} \mathrm{C}$. The lysates were incubated with $2 \mu \mathrm{g}$ of mouse anti-CD1d antibody (clone 51.1.3; a generous gift of S. Porcelli, Albert Einstein College of Medicine, Bronx, New York, USA) (46) which recognizes the $\beta-2$ complexed form of CD1d or rat anti-mouse CD1d (Clone 1B1; BD Biosciences - Pharmingen). After 1 hour of mixing at $4^{\circ} \mathrm{C}, 20 \mu \mathrm{l}$ of protein $\mathrm{A} / \mathrm{G}$ plus agarose beads (for the mouse antibody) or protein $\mathrm{G}$ beads (for the rat antibody; both from Santa Cruz Biotechnology Inc.) were then added to mix with the lysates overnight at $4^{\circ} \mathrm{C}$. The immunoprecipitation beads were then washed 4 times with cold PBS and resuspended in SDS loading buffer. The immunoprecipitates were then separated by SDS-PAGE (4-20\% Tris-HCl gradient gel; Bio-Rad Laboratories), and the proteins were transferred to nitrocellulose (Immobolion-P; 
Millipore). After blocking overnight in TBS, Tween20, and 5\% milk, the membrane was incubated for 1 hour with mouse anti-ubiquitin (N-19) antibody (Santa Cruz Biotechnology Inc.) preconjugated to HRP. After extensive washings ( 3 times for 15 minutes and 3 times for 5 minutes) with TBS, Tween 20 , and $5 \%$ milk, the antibodies were visualized with Luminol (Santa Cruz Biotechnology Inc.).

Dynamin dominant-negative experiments. BJAB cells were transfected with 24 $\mu \mathrm{g}$ of DNA in a 3:2:1 ratio consisting of PCR3.1 expressing the wild-type chimeric CD1d (Figure 5D), either empty or MIR-expressing PCR3.1, and PIRES2-EGFP with either a wild-type or a dominant-negative allele (K44E) of dynamin (41). The wild-type and K44E dynamin constructs were a kind gift of R.B. Vallee (Worcester Foundation for Experimental Biology, Shrewsbury, Massachusetts, USA). After 40 hours, the cells were stained, and surface levels of chimeric CD1d were measured by flow cytometry. The EGFP-expressing cells were gated upon and the levels of chimeric CD1d were analyzed.

Pulse-chase analysis. HepG2 cells (6-cm dish per time point) stable for the expression of MIR2 or lacZ as a control were used. Cells were preincubated with cysteine- and methionine-free DMEM (Sigma-Aldrich) supplemented with $10 \%$ (vol/vol) FCS and a penicillin-streptomycin mixture for 1 hour at $37^{\circ} \mathrm{C}$. The cells were labeled for 20 minutes with $400 \mu \mathrm{Ci}$ of $\mathrm{S}^{35}$ trans-label (ICN Biomedicals) in a final volume of $2 \mathrm{ml}$. After washes, cells were chased for the times indicated in Figure 6, B and C, in $50 \mathrm{ml}$ of complete MEM. At the end of chase periods, cells were lysed in $500 \mu \mathrm{l}$ of PBS containing $1 \%$ Nonidet P-40 and protease inhibitor mixture (Roche Applied Science) on ice for $30 \mathrm{~min}$. Nuclei and insoluble debris were removed by centrifugation. The remaining extract was incubated with $2 \mu \mathrm{g}$ of anti-CD $1 \mathrm{~d} \mathrm{mAb}$ or anti-MHC class I mAb (Clone W6/32; Caltag Laboratories) for 1 hour at $4{ }^{\circ} \mathrm{C}$. The mixture was supplemented with BSA to a final concentration of $1 \%$ and incubated overnight with $40 \mu \mathrm{l}$ of protein A/G beads (Santa Cruz Biotechnology Inc.) at $4^{\circ} \mathrm{C}$. Protein $\mathrm{A} / \mathrm{G}$ beads were washed 4 times with ice-cold PBS, and the bound proteins were analyzed by electrophoresis in SDS-PAGE (4-20\% Tris-HCl gradient gel; Bio-Rad Laboratories). Gels were dried and analyzed using a Storm 860 PhosphorImager (Molecular Dynamics). Chloroquine (Sigma-Aldrich) was added directly to the chase medium at the beginning of the chase period, from a 100x stock in PBS (final concentration, $10 \mu \mathrm{m}$ ).

\section{Acknowledgments}

This work was supported by the Howard Hughes Medical Institute and by an National Institute of General Medical Sciences pre-doctoral training grant (1R25GM56847; to D.J. Sanchez). We would like to thank L. Coscoy and J. Baron as well as other members of the D. Ganem lab for invaluable advice, and L. Lanier and M. Lodoen for their helpful insight and advice in the setup of the coculture system and IFN- $\gamma$ release assays. In addition, we thank J. Betchel, B. Glaunsinger, Y.T. Liu, and Y. Liang for culturing of the BCBL-1 cells. D. Ganem is an Investigator of the Howard Hughes Medical Institute.

Received for publication November 29, 2004, and accepted in revised form March 1, 2005.

Address correspondence to: Don Ganem, Department of Microbiology, University of California, San Francisco, Box 0552, Health Sciences West 1522, 513 Parnassus Avenue, San Francisco, California 94143-0552, USA. Phone: (415) 476-2826; Fax: (415) 476-0939; E-mail: ganem@cgl.ucsf.edu.
1. Katze, M.G., He, Y., and Gale, M., Jr. 2002. Viruses and interferon: a fight for supremacy. Nat. Rev. Immunol. 2:675-687.

2. French, A.R., and Yokoyama, W.M. 2003. Natural killer cells and viral infections. Curr. Opin. Immunol. 15:45-51.

3. Gumperz, J.E., and Brenner, M.B. 2001. CD1-specific T cells in microbial immunity. Curr. Opin. Immunol. 13:471-478.

4. Porcelli, S.A., and Modlin, R.L. 1999. The CD1 system: antigen-presenting molecules for $\mathrm{T}$ cell recognition of lipids and glycolipids. Annu. Rev. Immunol. 17:297-329.

5. Beckman, E.M., et al. 1996. CD1c restricts responses of mycobacteria-specific T cells. Evidence for antigen presentation by a second member of the human CD1 family. J. Immunol. 157:2795-2803.

6. Beckman, E.M., et al. 1994. Recognition of a lipid antigen by CD1-restricted alpha beta $+\mathrm{T}$ cells. Nature. 372:691-694.

7. Moody, D.B., et al. 1997. Structural requirements for glycolipid antigen recognition by CD1brestricted T cells. Science. 278:283-286.

8. Sieling, P.A., et al. 1995. CD1-restricted T cell recognition of microbial lipoglycan antigens. Science. 269:227-230.

9. Brigl, M., Bry, L., Kent, S.C., Gumperz, J.E., and Brenner, M.B. 2003. Mechanism of CD1d-restricted natural killer $\mathrm{T}$ cell activation during microbial infection. Nat. Immunol. 4:1230-1237.

10. Gumperz, J.E., et al. 2000. Murine CD1d-restricted $\mathrm{T}$ cell recognition of cellular lipids. Immunity. 12:211-221.

11. Bendelac, A., Rivera, M.N., Park, S.H., and Roark, J.H. 1997. Mouse CD1-specific NK1 T cells: development, specificity, and function. Annu. Rev. Immunol. 15:535-562.

12. Behar, S.M., and Cardell, S. 2000. Diverse CD1drestricted T cells: diverse phenotypes, and diverse functions. Semin. Immunol. 12:551-560.

13. Kawano, T., et al. 1997. CD1d-restricted and TCR- mediated activation of valpha14 NKT cells by glycosylceramides. Science. 278:1626-1629.

14. Baron, J.L., et al. 2002. Activation of a nonclassical NKT cell subset in a transgenic mouse model of hepatitis B virus infection. Immunity. 16:583-594.

15. Johnson, T.R., Hong, S., Van Kaer, L., Koezuka, Y., and Graham, B.S. 2002. NK T cells contribute to expansion of CD8(+) T cells and amplification of antiviral immune responses to respiratory syncytial virus. J. Virol. 76:4294-4303.

16. Grubor-Bauk, B., Simmons, A., Mayrhofer, G., and Speck, P.G. 2003. Impaired clearance of herpes simplex virus type 1 from mice lacking CD1d or NKT cells expressing the semivariant $\mathrm{V}$ alpha 14-J alpha 281 TCR. J. Immunol. 170:1430-1434.

17. Roberts, T.J., Lin, Y., Spence, P.M., Van Kaer, L., and Brutkiewicz, R.R. 2004. CD1d1-dependent control of the magnitude of an acute antiviral immune response. J. Immunol. 172:3454-3461.

18. Ploegh, H.L. 1998. Viral strategies of immune evasion. Science. 280:248-253.

19. Yewdell, J.W., and Hill, A.B. 2002. Viral interference with antigen presentation. Nat. Immunol. 3:1019-1025.

20. Chatterjee, M., Osborne, J., Bestetti, G., Chang, Y., and Moore, P.S. 2002. Viral IL-6-induced cell proliferation and immune evasion of interferon activity. Science. 298:1432-1435.

21. Zhu, F.X., King, S.M., Smith, E.J., Levy, D.E., and Yuan, Y. 2002. A Kaposi's sarcoma-associated herpesviral protein inhibits virus-mediated induction of type I interferon by blocking IRF-7 phosphorylation and nuclear accumulation. Proc. Natl. Acad. Sci. U. S. A. 99:5573-5578.

22. Eidson, K.M., Hobbs, W.E., Manning, B.J., Carlson, P., and DeLuca, N.A. 2002. Expression of herpes simplex virus ICP0 inhibits the induction of interferon-stimulated genes by viral infection. J. Virol. 76:2180-2191.

23. He, B., Gross, M., and Roizman, B. 1997. The gamma(1)34.5 protein of herpes simplex virus 1 complexes with protein phosphatase 1alpha to dephosphorylate the alpha subunit of the eukaryotic translation initiation factor 2 and preclude the shutoff of protein synthesis by doublestranded RNA-activated protein kinase. Proc. Natl. Acad. Sci. U. S. A. 94:843-848.

24. Lodoen, M., et al. 2003. NKG2D-mediated natural killer cell protection against cytomegalovirus is impaired by viral gp 40 modulation of retinoic acid early inducible 1 gene molecules. J. Exp. Med. 197:1245-1253.

25. Ganem, D. 1997. KSHV and Kaposi's sarcoma: the end of the beginning? Cell. 91:157-160.

26. Dourmishev, L.A., Dourmishev, A.L., Palmeri, D., Schwartz, R.A., and Lukac, D.M. 2003. Molecular genetics of Kaposi's sarcoma-associated herpesvirus (human herpesvirus-8) epidemiology and pathogenesis. Microbiol. Mol. Biol. Rev. 67:175-212.

27. Hengge, U.R., et al. 2002. Update on Kaposi's sarcoma and other HHV8 associated diseases. Part 2: pathogenesis, Castleman's disease, and pleural effusion lymphoma. Lancet. Infect. Dis. 2:344-352.

28. Coscoy, L., and Ganem, D. 2000. Kaposi's sarcomaassociated herpesvirus encodes two proteins that block cell surface display of MHC class I chains by enhancing their endocytosis. Proc. Natl. Acad. Sci. U. S. A. 97:8051-8056

29. Haque, M., et al. 2001. Major histocompatibility complex class I molecules are down-regulated at the cell surface by the K5 protein encoded by Kaposi's sarcoma-associated herpesvirus/human herpesvirus-8. J. Gen. Virol. 82:1175-1180.

30. Ishido, S., Wang, C., Lee, B.S., Cohen, G.B., and Jung, J.U. 2000. Downregulation of major histocompatibility complex class I molecules by Kaposi's sarcoma-associated herpesvirus $\mathrm{K} 3$ and $\mathrm{K} 5$ proteins. J. Virol. 74:5300-5309.

31. Stevenson, P.G., Efstathiou, S., Doherty, P.C., and Lehner, P.J. 2000. Inhibition of MHC class I-restricted antigen presentation by gamma 2-herpesviruses. Proc. Natl. Acad. Sci. U. S. A. 97:8455-8460. 
32. Coscoy, L., Sanchez, D.J., and Ganem, D. 2001. A novel class of herpesvirus-encoded membranebound E3 ubiquitin ligases regulates endocytosis of proteins involved in immune recognition. J. Cell Biol. 155:1265-1273.

33. Coscoy, L., and Ganem, D. 2001. A viral protein that selectively downregulates ICAM-1 and B7-2 and modulates $\mathrm{T}$ cell costimulation. J. Clin. Invest. 107:1599-1606.

34. Ishido, S., et al. 2000. Inhibition of natural killer cellmediated cytotoxicity by Kaposi's sarcoma-associated herpesvirus K5 protein. Immunity. 13:365-374.

35. Chang, J., Renne, R., Dittmer, D., and Ganem, D. 2000. Inflammatory cytokines and the reactivation of Kaposi's sarcoma-associated herpesvirus lytic replication. Virology. 266:17-25.

36. Sanchez, D.J., Coscoy, L., and Ganem, D. 2002. Functional organization of MIR2, a novel viral regulator of selective endocytosis. J. Biol. Chem. 277:6124-6130.

37. Kang, S.J., and Cresswell, P. 2002. Regulation of intracellular trafficking of human CD1d by association with MHC class II molecules. EMBO J. 21:1650-1660.

38. Boname,J.M., and Stevenson, P.G. 2001. MHC class I ubiquitination by a viral PHD/LAP finger protein. Immunity. 15:627-636.

39. Hewitt, E.W., et al. 2002. Ubiquitylation of MHC class I by the $\mathrm{K} 3$ viral protein signals internalization and TSG101-dependent degradation. EMBOJ. 21:2418-2429.

40. Katzmann, D.J., Odorizzi, G., and Emr, S.D. 2002. Receptor downregulation and multivesicular-body sorting. Nat. Rev. Mol. Cell Biol. 3:893-905.

41. Herskovits, J.S., Burgess, C.C., Obar, R.A., and Vallee, R.B. 1993. Effects of mutant rat dynamin on endocytosis. J. Cell Biol. 122:565-578.

42. Tomescu, C., Law, W.K., and Kedes, D.H. 2003. Surface downregulation of major histocompatibility complex class I, PE-CAM, and ICAM-1 following de novo infection of endothelial cells with Kaposi's sarcoma-associated herpesvirus. J. Virol. 77:9669-9684.

43. Krmpotic, A., et al. 2002. MCMV glycoprotein gp40 confers virus resistance to CD8+ T cells and NK cells in vivo. Nat. Immunol. 3:529-535.

44. Nishimura, T., et al. 2000. The interface between innate and acquired immunity: glycolipid antigen presentation by CD1d-expressing dendritic cells to NKT cells induces the differentiation of antigenspecific cytotoxic T lymphocytes. Int. Immunol. 12:987-994.

45. Carnaud, C., et al. 1999. Cutting edge: Crosstalk between cells of the innate immune system NKT cells rapidly activate NK cells. J. Immunol. 163:4647-4650.

46. Chiu, N.M., Chun, T., Fay, M., Mandal, M., and Wang, C.R. 1999. The majority of H2-M3 is retained intracellularly in a peptide-receptive state and traffics to the cell surface in the presence of $\mathrm{N}$-formylated peptides. J. Exp. Med. 190:423-434.

47. Sriram, V., et al. 2002. Inhibition of glycolipid shedding rescues recognition of a CD1+ T cell lymphoma by natural killer T (NKT) cells. Proc. Natl. Acad. Sci. U. S. A. 99:8197-8202.

48. Castano, A.R., et al. 1995. Peptide binding and presentation by mouse CD1. Science. 269:223-226.
49. Sugita, M., et al. 2002. Failure of trafficking and antigen presentation by CD1 in AP-3-deficient cells. Immunity 16:697-706.

50. Elewaut, D., et al. 2003. The adaptor protein AP-3 is required for CD1d-mediated antigen presentation of glycosphingolipids and development of Valpha14i NKT cells. J. Exp. Med. 198:1133-1146.

51. Cernadas, M., et al. 2003. Lysosomal localization of murine CD1d mediated by AP- 3 is necessary for NK T cell development. J. Immunol. 171:4149-4155.

52. Chiu, Y.H., et al. 1999. Distinct subsets of CD1drestricted $\mathrm{T}$ cells recognize self-antigens loaded in different cellular compartments. J. Exp. Med. 189:103-110.

53. Roberts, T.J., et al. 2002. Recycling CD1d1 molecules present endogenous antigens processed in an endocytic compartment to NKT cells. J. Immunol. 168:5409-5414.

54. Prigozy, T.I., et al. 2001. Glycolipid antigen processing for presentation by CD1d molecules. Science. 291:664-667.

55. Zhou, D., et al. 2004. Editing of Cd1d-bound lipid antigens by endosomal lipid transfer proteins. Science. 303:523-527.

56. Jayawardena-Wolf, J., Benlagha, K., Chiu, Y.H., Mehr, R., and Bendelac, A. 2001. CD1d endosomal trafficking is independently regulated by an intrinsic CD1d-encoded tyrosine motif and by the invariant chain. Immunity. 15:897-908.

57. Gumperz, J.E., Miyake, S., Yamamura, T., and Brenner, M.B. 2002. Functionally distinct subsets of CD1d-restricted natural killer T cells revealed by CD1d tetramer staining. J. Exp. Med. 195:625-636. 